\title{
Analytical generation model of surface electromyogram for multi-layer volume conductors
}

\author{
L. Mesin \\ LISiN, Laboratorio di Ingegneria del Sistema Neuromuscolare, \\ Dipartimento di Elettronica, Politecnico di Torino, Torino, Italy
}

\begin{abstract}
Surface electromyogram (EMG) is a bio-electrical signal detected non-invasively (over the skin) and reflecting features of the bio-electrical sources associated to muscle contraction. Surface EMG signal modelling has important applications in the interpretation of experimental EMG data, for a deeper understanding of the physiological mechanisms of muscle contraction. This study addresses the issue of introducing a general analytical method to study multi layer volume conductors (i.e., to solve a Poisson problem on domains constituted by multiple layered sub-domains, with different electrical conductivities). The method provides the solution to a multi plane layer problem, once the solutions of the homogeneous Dirichlet and Neumann problems in a one layer volume conductor are available. The same method can be applied to cylindrical layer volume conductors (some indications are given). It is applied to add a fat layer over a bipinnate muscle layer (i.e., a muscle which has two groups of fibres with two orientations) which was analytically studied in the literature (considering only the muscle layer). The study provides an implementation of the results in a complete surface EMG generation model (including finite length fibres), and shows representative results of the application of the model proposed.

Poisson equation models different physical and biological situations. Thus, the method introduced can find applications in other fields of interest for the applied sciences.

Keywords: Poisson equation, multi layer volume conductor, Fourier transform, surface electromyogram.
\end{abstract}




\section{Introduction}

The contraction of a muscle fibre is primed by the intra-cellular action potential (IAP), which is generated at the end plate, and propagates along the fibre toward the tendons, where it extinguishes. The IAP is a bio-electric source which determines a potential in the surrounding physiological tissues, endowed with electrical conductive properties. The non invasive detection of such a potential (on the skin) is called surface electromyography and the detected signal is the surface electromyogram (sEMG). The simulation of sEMG is important for a deeper understanding of the physiological mechanisms of muscle contraction (Kleine et al. [13]), through the estimation of physiological variables (inverse problem) (van Oosterom [25]). Furthermore, it can help in the choice of the detection system (Dimitrov et al. [3]; Farina et al. [7]), in the interpretation of experimental results (Dimitrova and Dimitrov [4]; Roeleveld et al. [22]), and for didactic purposes (Merletti et al. [16, 17]; Stegeman et al. [24]). A sEMG model is built by the following steps: 1) description of the source (i.e., generation, propagation, and extinction of the IAP); 2) mathematical description of the volume conductor; 3 ) modelisation of the detection system (spatial arrangement, shape and size of the electrodes) (for points 1), 2), 3) see Farina [9] and the bibliography therein); 4) description of the spatial and temporal recruitment strategy of the motor units (MUs), which are the basic functional blocks of the neuromuscular system (Fuglevand et al. [10]).

The volume conductor can be studied either analytically or numerically (Lowery et al. [14]; Schneider et al. [23]; Farina et al. [9]; Mesin et al. [21]). Analytical solutions can be obtained only in specific cases, while numerical methods are necessary when considering more complex conditions. Nevertheless, analytical solutions are valuable, as they allow one to determine the theoretical dependence of the solution on specific parameters of the system, to check the accuracy of numerical methods, and to reduce the computational time. Some examples of volume conductors, for which analytical generation models of sEMG are available, are the following: isotropic, homogeneous, infinite medium (Clark and Plonsey [2]); multi layer models for planar (Farina and Merletti [5]) and cylindrical (Gootzen et al. [11]; Block et al. [1]; Farina et al. [8]) volume conductors; volume conductors with local (Mesin and Farina [19]) and with distributed (Mesin and Farina [20]) in-homogeneity; bipinnate muscle (Mesin and Farina [18]); muscle with fibres inclined with respect to the detection surface (Mesin and Farina [18]).

In this work, I present a general method to add a layer (or more layers, by iteration) to a volume conductor for which a solution is known. The method is checked by comparison with the known solution for the case of homogeneous, plane, multi layer tissues (Farina et al. [5]). It is then applied to a non homogeneous single layer model of a bi-pinnate muscle, providing the analytical solution for a new model of multi layer bi-pinnate muscle. A general discussion on further possible fields of application is finally provided. 


\section{Methods}

An analytical method for studying multi layer volume conductors is introduced. The method is first tested on a model for which an analytical solution (obtained by a different method) is known, and then is applied to study a new volume conductor model, i.e. a multi layer model of a bi-pinnate muscle.

\subsection{Mathematical problem for a volume conductor}

Electrical field problems in physiology can be considered (within a good approximation) as quasi-static, Heringa et al. [12]. Thus, the physiological tissue can be described as a volume conductor. In these conditions, the electrical potential satisfies Poisson equation:

$$
-\nabla \cdot(\underline{\underline{\sigma}} \nabla \varphi)=I
$$

where $\varphi$ is the potential $(V), I$ the current density source $\left(A / \mathrm{m}^{3}\right)$, and $\underline{\underline{\sigma}}$ the conductivity tensor $(S / m)$.

A mathematical problem is obtained by adding boundary conditions to eqn (1). Usually a physiological volume conductor is considered insulated (i.e., the conductivity of the surrounding medium, e.g. air, is neglected), which means that a homogeneous Neumann problem is written. If more layers (with different conductivity) are studied, interface conditions of continuity of the potential and of its flux (i.e., the current density vector in the direction normal to the interface surface) are considered.

\subsection{General method for multi layer volume conductors}

The proposed method allows to add a plane layer over a volume conductor for which the solution of the homogeneous Neumann problem and the normal derivative of the solution of the homogeneous Dirichlet problem are available (it is enough to know such solutions at the interface surface). It is worth noticing that for a one plane layer model, both homogeneous Neumann and Dirichlet solutions can be obtained from the solution for the infinite medium, by image theorem. This means that knowing the solution for the problem in the infinite medium is sufficient to solve a multi layer problem.

Let us consider the solution of the homogeneous Neumann problem $\varphi_{N}$ and of the homogeneous Dirichlet problem $\varphi_{D}$ in an arbitrary semi-infinite medium (infinite in the $x, z$ direction, and defined on $y<0$ ), which will be considered as the muscle layer

$$
\left\{\begin{array} { l } 
{ - \nabla \cdot ( \underline { \sigma } \nabla \varphi _ { N } ) = \delta ( x ) \delta ( y - y _ { 0 } ) \delta ( z ) } \\
{ \frac { \partial \varphi _ { N } } { \partial y } | _ { y = 0 } = 0 }
\end{array} \quad \left\{\begin{array}{l}
-\nabla \cdot\left(\underline{\sigma} \nabla \varphi_{D}\right)=\delta(x) \delta\left(y-y_{0}\right) \delta(z) \\
\left.\varphi_{D}\right|_{y=0}=0
\end{array}\right.\right.
$$


where $\delta$ indicates the Dirac delta function and $\underline{\underline{\sigma}}=\sigma_{T}(\hat{x} \hat{x}+\hat{y} \hat{y})+\sigma_{L} \hat{z} \hat{z}$ (anisotropic conductivity tensor), where $\sigma_{T}$ is the conductivity in the transversal direction with respect to the direction of propagation $\hat{z}$, and $\sigma_{L}$ is the longitudinal conductivity. Vanishing condition at $y \rightarrow-\infty$ is further imposed.

The method is based on the following ansatz for the solution of a general boundary value problem

$$
\varphi=a(x, z) * \varphi_{N}(x, y, z)+b(x, z) * \varphi_{D}(x, y, z)
$$

which means that the solution can be expressed in terms of $\varphi_{N}$ and $\varphi_{D}$, by a linear convolutive mixture with kernels $a(x, z)$ and $b(x, z)$, still to be determined. Substituting eqn (3) in eqn (2), the equation is satisfied with the following condition for the kernels

$$
a(x, z)+b(x, z)=\delta(x) \delta(z)
$$

The particular expression for the kernels can be obtained by further imposing the boundary conditions. In this work, the general expression (3) is used to fit interface conditions between two media.

For the application to sEMG simulation, I consider the case of a semi-infinite muscle layer (just studied) and a homogeneous layer defined on $0<y<d$, placed over the muscle layer (which could model a skin, subcutaneous, or fat layer; in the following, I will refer to such a layer as the fat layer). The equation to be solved in the fat layer is Laplace equation. It can be solved in the Fourier domain, transforming from the $x, z$ to the $k_{x}, k_{z}$ variables

$$
\frac{d^{2} \hat{\varphi}_{S}}{d y^{2}}=k_{y}^{2} \hat{\varphi}_{S}
$$

where $k_{y}=\sqrt{k_{x}{ }^{2}+k_{z}{ }^{2}}$, and $\hat{\varphi}_{S}=\hat{\varphi}_{S}\left(y ; k_{x}, k_{z}\right)$, where the spatial frequencies are considered as parameters of the set of ordinary differential equations (ODE) in eqn (5). Solving eqn (5) the following general solution is obtained

$$
\hat{\varphi}_{S}=C e^{k_{y} y}+C_{1} e^{-k_{y} y} \quad 0<y<d
$$

Imposing Neumann homogeneous condition at $y=d$ yields the following expression

$$
\hat{\varphi}_{S}=C\left(e^{k_{y} y-2 d k_{y}}+e^{-k_{y} y}\right) \quad 0<y<d
$$

which has a degree of freedom expressed by the constant $C$, which depends on the spatial frequencies $k_{x}, k_{z}$. Such a degree of freedom can be used to fit the interface conditions with the muscle layer. By imposing continuity of the potential and of its flux at $y=0$ and eqn (4) in the transformed domain, the following system of 3 equations in 3 unknowns $(A, B, C$, where $A$ and $B$ are the Fourier transforms of $a$ and $b$, respectively) is obtained 


$$
\left\{\begin{array}{l}
A+B=1 \\
\left.A \hat{\varphi}_{N}\right|_{y=0}=\left.\hat{\varphi}_{S}\right|_{y=0} \\
\left.\sigma_{T} B \frac{\partial \hat{\varphi}_{D}}{\partial y}\right|_{y=0}=\left.\sigma \frac{\partial \hat{\varphi}_{S}}{\partial y}\right|_{y=0}
\end{array}\right.
$$

The following expressions for the unknowns $A, B, C$ are obtained

$$
\begin{gathered}
A=\frac{\left.\sigma_{T} \frac{\partial \hat{\varphi}_{D}}{\partial y}\right|_{y=0}\left(1+e^{2 k_{y} d}\right)}{\left.\sigma k_{y} \hat{\varphi}_{N}\right|_{y=0}\left(1-e^{2 k_{y} d}\right)+\left.\sigma_{T} \frac{\partial \hat{\varphi}_{D}}{\partial y}\right|_{y=0}\left(1+e^{2 k_{y} d}\right)} \\
B=\frac{\left.\sigma k_{y} \hat{\varphi_{N}}\right|_{y=0}\left(1-e^{2 k_{y} d}\right)}{\left.\sigma_{k_{y}} \hat{\varphi}_{N}\right|_{y=0}\left(1-e^{2 k_{y} d}\right)+\left.\sigma_{T} \frac{\partial \hat{\varphi}_{D}}{\partial y}\right|_{y=0}\left(1+e^{2 k_{y} d}\right)} \\
C=\frac{\left.\left.\sigma_{T} \hat{\varphi}_{N}\right|_{y=0} \frac{\partial \hat{\varphi}_{D}}{\partial y}\right|_{y=0}}{\left.\sigma k_{y} \hat{\varphi}_{N}\right|_{y=0}\left(1-e^{2 k_{y} d}\right)+\left.\sigma_{T} \frac{\partial \hat{\varphi_{D}}}{\partial y}\right|_{y=0}\left(1+e^{2 k_{y} d}\right)}
\end{gathered}
$$

To determine the surface potential (for an insulated two layer model) only the expression for $C$ is needed.

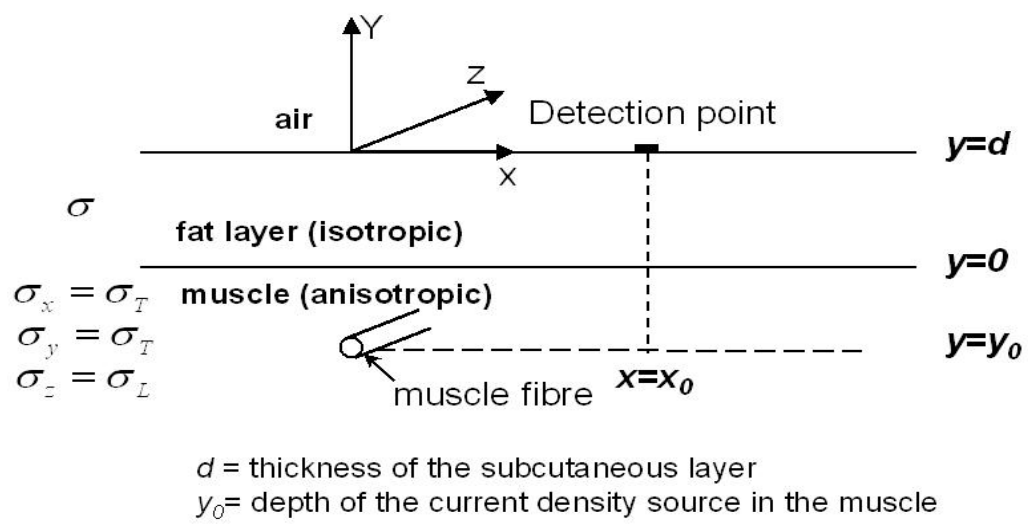

Figure 1: Non-homogeneous (layered), an-isotropic volume conductor model constituted by muscle (an-isotropic) and fat (isotropic) layers. The volume conductor is infinite in $x$ and $z$ directions and semi-infinite in $y$ direction (infinite in the negative direction, bounded at $y=d$ ). The $z$ direction is that of the muscle fibres (along which the action potentials propagate).

Once the potential at the surface of the fat layer is obtained, it is possible, if needed, to iterate the method for adding another layer. To do that, also the 
solution of the homogeneous Dirichlet problem is needed (eqn (2)). Considering eqn (6) and imposing homogeneous Dirichlet condition at $y=d$, the following general solution in the fat layer is obtained

$$
\hat{\varphi}_{S}=C\left(-e^{k_{y} y-2 d k_{y}}+e^{-k_{y} y}\right) \quad 0<y<d
$$

Solving system (8) for this new expression of the solution in the fat layer yields

$$
A=\frac{\left.\sigma_{T} \frac{\partial \hat{\varphi}_{D}}{\partial y}\right|_{y=0}\left(1-e^{2 k_{y} d}\right)}{\left.\sigma k_{y} \hat{\varphi}_{N}\right|_{y=0}\left(1+e^{2 k_{y} d}\right)+\left.\sigma_{T} \frac{\partial \hat{\varphi}_{D}}{\partial y}\right|_{y=0}\left(1-e^{2 k_{y} d}\right)}
$$

In summary: the expression reported in eqn (7) substituting $C$ from (9.III) solves the homogeneous Neumann problem in the fat layer; the expression reported in eqn (10) substituting $C$ from (11.III) solves the homogeneous Dirichlet problem. These two solutions can be considered as the Fourier transforms of $\varphi_{N}$ and $\varphi_{D}$ (respectively) in eqn (2) for a two layer model. Thus, the analysis can be applied iteratively, adding as many layers as needed.

The extension of the method to a cylindrical geometry is lengthy, but straightforward. The general solutions in eqns (7) and (10) should be replaced by those equivalent for a cylindrical layer (in terms of a series of modified Bessel functions, see Farina et al. [8]). System (8) can then be solved in the cylindrical co-ordinates system, giving expressions equivalent to those reported in eqns (9) and (11).

Another extension of the method can be obtained. Suppose the impulse responses correspondent to homogeneous Neumann and Dirichlet conditions at the interface surface are known for two media. Let us indicate by 1 and 2 such two media, adding apex or pedex 1 or 2 to the expressions reported in (2) and (3) to indicate the corresponding solutions and kernels. Suppose we want to solve the two layer problem, with the impulse located in medium 2. Laplace equation is thus considered in medium 1. The condition corresponding to (4) for the general medium 1, satisfying Laplace equation, is the following

$$
a_{1}(x, z)+b_{1}(x, z)=0
$$

Working again in the Fourier transformed domain (transforming the $x$ and $z$ variables into $\left.k_{x}, k_{z}\right)$, the following system of 4 equations in 4 unknowns $\left(A_{l}, B_{l}\right.$, $\left.A_{2}, B_{2}\right)$ is obtained by imposing conditions (4) and (4'), and the interface conditions 


$$
\left\{\begin{array}{l}
A_{1}+B_{1}=0 \\
A_{2}+B_{2}=1 \\
\left.A_{1} \hat{\varphi}_{N}^{1}\right|_{y=0}=\left.A_{2} \hat{\varphi}_{N}^{2}\right|_{y=0} \\
\left.\sigma_{T 1} B_{1} \frac{\partial \hat{\varphi}_{D}^{1}}{\partial y}\right|_{y=0}=\left.\sigma_{T 2} B_{2} \frac{\partial \hat{\varphi}_{D}^{2}}{\partial y}\right|_{y=0}
\end{array}\right.
$$

The solution of system (12) is the following

$$
\begin{gathered}
A_{1}=-\frac{\left.\left.\sigma_{T 2} \hat{\varphi}_{N}^{2}\right|_{y=0} \frac{\partial \hat{\varphi}_{D}^{2}}{\partial y}\right|_{y=0}}{\left.\left.\sigma_{T 1} \frac{\partial \hat{\varphi}_{D}^{1}}{\partial y}\right|_{y=0} \hat{\varphi}_{N}^{2}\right|_{y=0}-\left.\left.\sigma_{T 2} \frac{\partial \hat{\varphi}_{D}^{2}}{\partial y}\right|_{y=0} \hat{\varphi}_{N}^{1}\right|_{y=0}} \quad B_{1}=-A_{1} \\
A_{2}=-\frac{\left.\left.\sigma_{T 2} \hat{\varphi}_{N}^{1}\right|_{y=0} \frac{\partial \hat{\varphi}_{D}^{2}}{\partial y}\right|_{y=0}}{\left.\left.\sigma_{T 1} \frac{\partial \hat{\varphi}_{D}^{1}}{\partial y}\right|_{y=0} \hat{\varphi}_{N}^{2}\right|_{y=0}-\left.\left.\sigma_{T 2} \frac{\partial \hat{\varphi}_{D}^{2}}{\partial y}\right|_{y=0} \hat{\varphi}_{N}^{1}\right|_{y=0}} \quad B_{2}=1-A_{2}
\end{gathered}
$$

The solution reported in (13) allows to interface (with a plane contact surface) general media for which homogeneous Neumann and Dirichlet problems can be solved.

\subsubsection{Example of application to a plane homogeneous layer model}

In this section, the simplest multi layer volume conductor is considered. It is a two plane layer model, obtained by putting into contact two homogeneous media, and an-isotropic muscle layer and an isotropic fat layer, Farina et al. [5], see Figure 1. The solution for the configuration described in Figure 1 is known and may therefore provide a golden standard for testing the method introduced in the previous section.

By transforming the $x, z$ variables into $k_{x}, k_{z}$, the following equations are obtained in the fat and muscle layers, respectively

$$
\sigma \frac{d^{2} \hat{\varphi}_{F}}{d y^{2}}=k_{y}^{2} \hat{\varphi}_{F} \quad \sigma_{T} \frac{d^{2} \hat{\varphi}_{M}}{d y^{2}}=k_{y a}^{2} \hat{\varphi}_{M}-\delta\left(y-y_{0}\right)
$$

where $\hat{\varphi}_{F}$ and $\hat{\varphi}_{M}$ are the Fourier transforms of the solutions in the fat and muscle layers, respectively, and $k_{y}=\sqrt{{k_{x}{ }^{2}+k_{z}{ }^{2}}}, k_{y a}=\sqrt{k_{x}{ }^{2}+R_{a} k_{z}{ }^{2}}$, being $R_{a}=\frac{\sigma_{L}}{\sigma_{T}}$ the an-isotropy ratio. The solution is obtained by separating the muscle in two domains, $\mathrm{y}<\mathrm{y}_{0}$ and $\mathrm{y}>\mathrm{y}_{0}$ (Farina [8]), writing the following solutions of the homogeneous equations

$$
\begin{array}{ll}
\hat{\varphi}_{F}=C e^{k_{y} y}+C_{1} e^{-k_{y} y} & 0<y<d \\
\hat{\varphi}_{M}=A_{2} e^{k_{y} y}+B_{2} e^{-k_{y} y} & y_{0}<y<0 \\
\hat{\varphi}_{M}=A_{1} e^{k_{y} y} \quad y<y_{0} &
\end{array}
$$


and imposing the following boundary and interface conditions

$$
\left\{\begin{array}{l}
\left.\frac{d \hat{\varphi}_{F}}{d y}\right|_{y=d}=0 \\
\left.\hat{\varphi}_{F}\right|_{y=0}=\left.\hat{\varphi}_{M}\right|_{y=0} \\
\left.\sigma \frac{d \hat{\varphi}_{F}}{d y}\right|_{y=0}=\left.\sigma_{T} \frac{d \hat{\varphi}_{M}}{d y}\right|_{y=0} \\
\left.\hat{\varphi}_{M}\right|_{y=y_{0}^{+}}=\left.\hat{\varphi}_{M}\right|_{y=y_{0}{ }^{-}}=\left.\sigma_{T} \frac{d \hat{\varphi}_{M}}{d y}\right|_{y=y_{0}^{-}}-1
\end{array}\right.
$$

where the discontinuity of the flux imposed by the impulse source was considered in eqn (16.V). System (16) consists in 5 equations in the 5 unknowns $A_{1}, A_{2}, B_{2}, C, C_{1}$. By solving the system, the following expression for the solution in the fat layer is obtained

$$
\hat{\varphi}_{F}=\frac{e^{k_{y a} y_{0}} \cosh \left(k_{y}\left(d-y_{0}\right)\right)}{k_{y} \sigma \sinh \left(k_{y} d\right)+k_{y a} \sigma_{T} \cosh \left(k_{y} d\right)}
$$

Let us apply now the proposed method. First, I need the solution of the homogeneous Neumann and Dirichlet problems (2) in the semi-infinite muscle layer. The solution in the infinite muscle layer can be obtained by considering the homogeneous solutions in $y<y_{0}$ and $y>y_{0}$

$$
\begin{array}{ll}
\hat{\varphi}_{M}=B_{2} e^{-k_{y} y} & y_{0}<y \\
\hat{\varphi}_{M}=A_{1} e^{k_{y} y} & y<y_{0}
\end{array}
$$

and imposing the following interface conditions at $y=y_{0}$

$$
\left\{\begin{array}{l}
\left.\hat{\varphi}_{M}\right|_{y=y_{0}{ }^{+}}=\left.\hat{\varphi}_{M}\right|_{y=y_{0}{ }^{-}}=\left.\sigma_{T} \frac{d \hat{\varphi}_{M}}{d y}\right|_{y=y_{0}{ }^{-}}-1 \\
\left.\sigma_{T} \frac{d \hat{\varphi}_{M}}{d y}\right|_{y=y_{0}{ }^{+}}=1
\end{array}\right.
$$

The following impulse response in the infinite muscle is obtained in the domain $y>y_{0}$

$$
\hat{\varphi}_{M}=\frac{e^{-k y a\left(y-y_{0}\right)}}{2 \sigma_{T} k_{y a}}
$$

It is possible to write system (8) by identifying the following quantities

$$
\left\{\begin{array}{l}
\left.\hat{\varphi}_{1}\right|_{y=0}=\left.2 \hat{\varphi}_{M}\right|_{y=0} \\
\left.\frac{\partial \hat{\varphi}_{2}}{\partial y}\right|_{y=0}=\left.2 \frac{\partial \hat{\varphi}_{M}}{\partial y}\right|_{y=0}
\end{array}\right.
$$


where the image theorem was applied to the infinite muscle solution. With these identifications, the term $C$ in (9.III) can be substituted in the general homogeneous Neumann solution (7) which gives the solution in the fat layer. The same expression reported in eqn (17) is obtained, verifying the identity of the solutions obtained with the two different approaches.

Also the general method reported in eqn (12) and (13) gives the same result. In such a case, two solutions correspondent to two impulse responses (relative to homogeneous Neumann and Dirichlet conditions imposed at $y=0$ ), one in the muscle and the other in the fat layer, are first obtained. Condition (4') allows one to place arbitrarily the impulse in the fat layer, without any trace of such an arbitrary location point in the final solution.

\subsubsection{Application to a non homogeneous model: the bi-pinnate muscle}

The problem of a plane insulated bi-pinnate muscle was studied in Mesin and Farina [18]. The solution was obtained in the Fourier two dimensional ( $x, z$ directions) transformed domain for a set of planes along the $y$ variable (see Figure 2).

Transforming from the $y$ to the $k_{y}$ variable, the solution (for the infinite muscle) is obtained as a function of the spatial frequencies $k_{x}, k_{y}, k_{z}$. Antitransforming the $k_{y}$ variable at $y=0$, the (Fourier transformed) surface potential at $y=0$ is obtained as a function of $k_{x}, k_{z}$. The normal derivative at the surface $y=0$ can be evaluated by inverting at $y=0$ the transformed solution (expressed as a function of the spatial frequencies $k_{x}, k_{y}, k_{z}$,) multiplied by $j k_{y}$. Multiplying by 2 the solution and its normal derivative at $y=0$, the solution of the homogeneous Neumann problem $\left.\hat{\varphi}_{N}\right|_{y=0}$ (already reported in Mesin [18]) and the normal derivative of the solution of the homogeneous Dirichlet problem $\left.\frac{\partial \hat{\varphi}_{D}}{\partial y}\right|_{y=0}$ are

obtained (by image theorem). These represent all the information needed for the method presented in Section 2.2 to solve a 2 layer problem. It is then possible to add a fat layer above the non homogeneous bi-pinnate muscle layer. The potential in the fat layer (with insulated condition at $y=d$ ) is given by eqn (7) substituting the term $C$ from (9.III).

The impulse responses for a one layer (muscle) and a two layer (muscle and fat) volume conductor are shown in Figure 3. The filtering effect of the homogeneous fat layer determines a reduction in amplitude and a more diffused surface potential, as expected.

The surface single fibre action potential (SFAP) can be obtained by simulating the generation, propagation, and extinction of tripole sources (McGill and Huynh [15], Merletti et al. [16]). In Figure 4 some representative examples of SFAPs, detected by 8 monopolar channels, are shown. The SFAPs relative to 4 fibres at $2 \mathrm{~mm}$ and 4 fibres at $5 \mathrm{~mm}$ depth within the muscle were simulated. The representative simulations show the decay of amplitude and the increased diffusion of the propagating component of the SFAPs increasing depth, and considering a two layer instead of an only muscle model. Non propagating 
components, related to the extinction of the tripole source at the tendons, are less affected by the anatomy, and have approximately constant amplitude on different channels. Transversal displacements of the fibre in the left and right directions with respect to the detection system (Fibre 1 and 3, respectively) is not only related to a decay of amplitude and to an increased diffusion of the propagating component. Indeed, we can note that the SFAPs related to Fibre 1 and 3 are very different, as the positions of the innervation zone and of the tendons with respect to the detection system change completely. This is an important difference between muscles with pinnate and with straight fibres. Indeed, in the case of muscle constituted by straight fibres the orthogonal projections of the positions of the end plate and of the tendons on the line through the detection channels is (ideally) constant considering different fibres moving transversely with respect to the detection array. This is not the case for pinnate muscles. Moreover, in a bipinnate muscle, when a detection array is placed over one bundle of fibres, there is a cross talk from the other bundle (Fibre 4 in Figure 4). Such signals change shape and amplitude between channels.

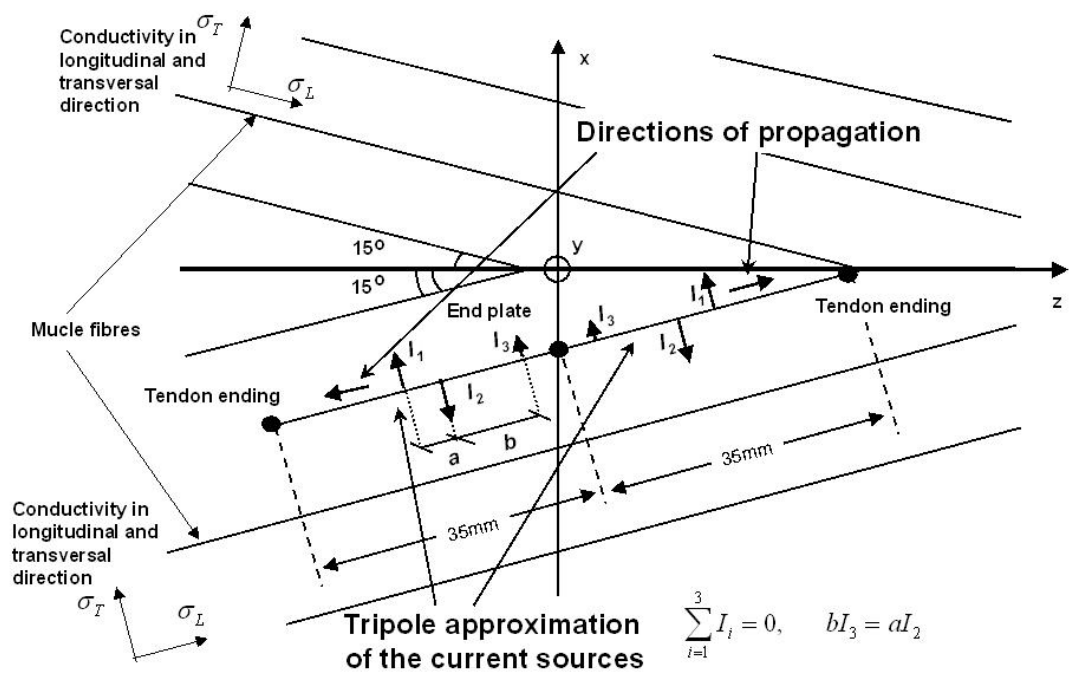

Figure 2: Non-homogeneous an-isotropic model of bi-pinnate muscle. The reported data are those used for the representative simulations reported in the text. The anatomical parameters chosen describe a rectus femuris muscle. The sources are modelled by current tripoles (impulse amplitudes: $I_{1}=24.6 \mathrm{~A} / \mathrm{m}^{2}, I_{2}=-35.4 \mathrm{~A} / \mathrm{m}^{2}, I_{3}=10.8 \mathrm{~A} / \mathrm{m}^{2}$; distances between poles: $a=2.1 \mathrm{~mm}, b=4.8 \mathrm{~mm}$, McGill and Huynh [15], Merletti [16]).

All the shape perturbations of the propagating potentials and the changes in direction of propagation can affect the estimation of conduction velocity ( $\mathrm{CV}$, 
physiological values between 3 and $5 \mathrm{~m} / \mathrm{s}$ ), which is one of the most important parameter to be extracted from sEMG signals, giving indications on muscle constitution and fatigue (see Farina and Merletti [6] for a recent review on CV estimation methods).
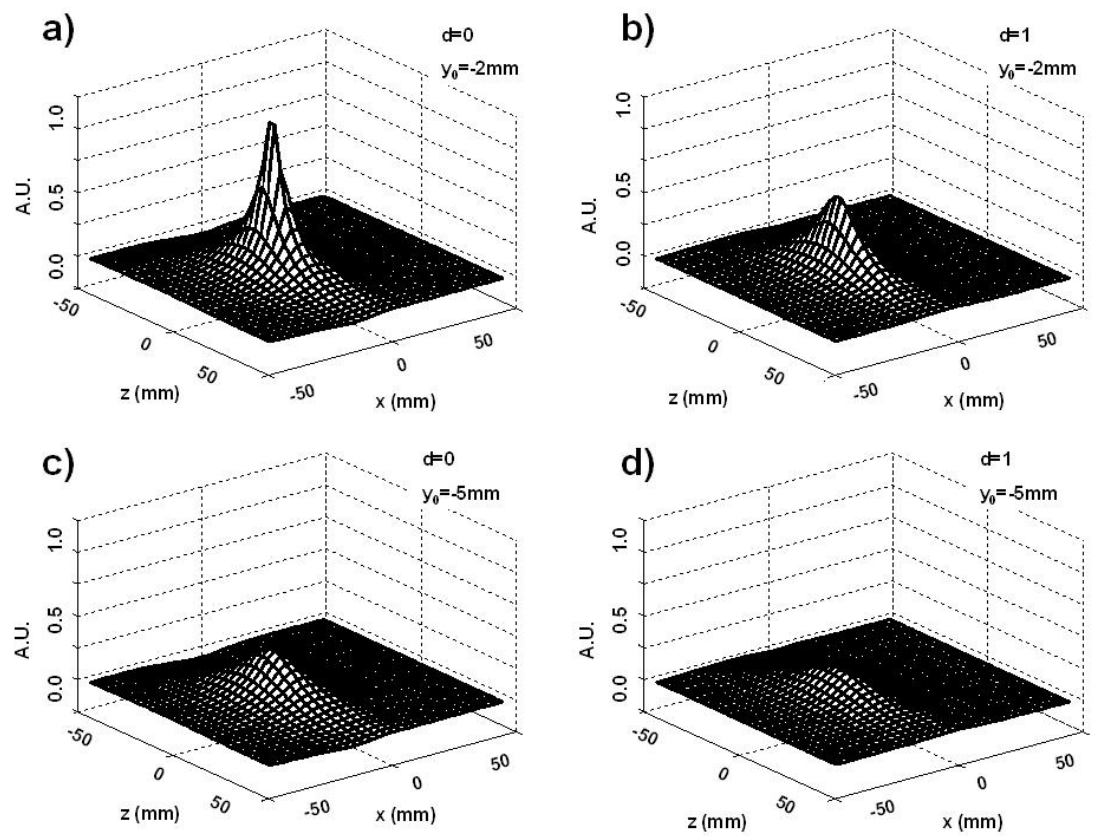

Figure 3: Impulse responses associated to impulses at $2 \mathrm{~mm}$ a), b) and $5 \mathrm{~mm} \mathrm{c}$ ), d) depth within the muscle, at $10 \mathrm{~mm}$ distance from the pinnation plane in the $x$ direction. A one layer (only muscle) model was used for a) and c), with $\sigma_{T}=0.1 \mathrm{~S} / \mathrm{m}, \sigma_{L}=0.5 \mathrm{~S} / \mathrm{m}$; a two layer model was considered for $b$ ) and d), placing over the muscle a plane fat layer, $1 \mathrm{~mm}$ thick, with $\sigma=0.05 \mathrm{~S} / \mathrm{m}$. Impulse responses are shown in arbitrary units (A.U.). Discretisation of the variables $k_{x}, k_{y}, k_{z}$ was 256 points. Maximum spatial frequency for $k_{x}, k_{z}$ corresponds to a temporal frequency of $4096 \mathrm{~Hz}$ for a conduction velocity of $4 \mathrm{~m} / \mathrm{s}$ (see Farina [8]). Maximum spatial frequency for $k_{y}$ corresponds to a discretisation of $0.1 \mathrm{~mm}$ in depth (the choice of 256 discretisation points makes negligible the aliasing for the maximal considered depth of $5 \mathrm{~mm}$ within the muscle). 

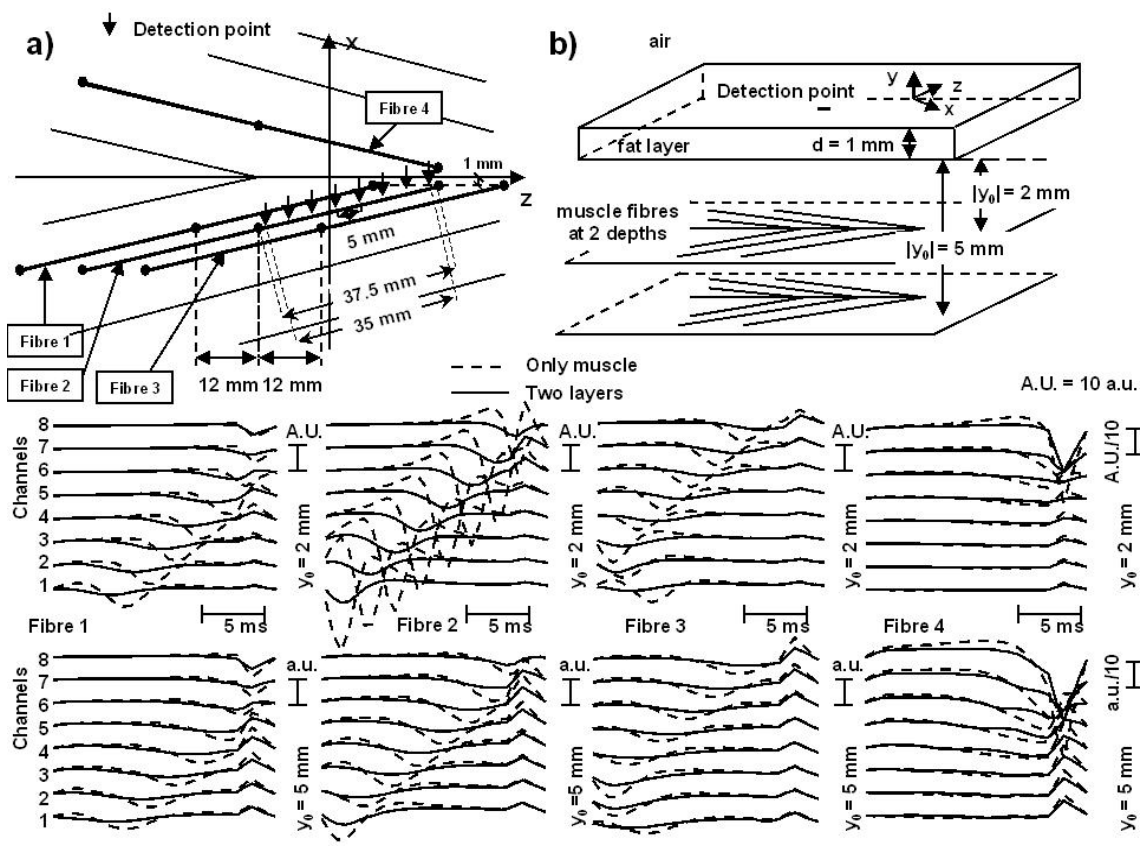

Figure 4: Monopolar single fibre action potentials relative to 4 fibres at $2 \mathrm{~mm}$ and 4 fibres at $5 \mathrm{~mm}$ depth within the muscle (the same names [fibre $1,2,3,4]$ are given to both the 4 fibres on the planes $y=-2 \mathrm{~mm}$ and $y=-5 \mathrm{~mm}$ ). Both a section of the muscle a) and a representation of a section along the depth direction $b$ ) are shown. The electrode array is above and parallel to fibre 2. Fibre 1 and 3 are parallel to fibre 2 and displaced from it of $12 \mathrm{~mm}$ in the $z$ direction. Fibre 4 is on the opposite side of the pinnation line.

\section{Discussion and conclusions}

Simulation of sEMG is important for testing algorithms and for a deeper understanding of muscle contraction. This paper introduces a new method for solving Poisson problems in multi layer plane domains (also cylindrical domains can be studied, by adapting the method to the geometry). The implementation of the results in a sEMG generation model for a bi-pinnate muscle covered by a fat layer provides representative simulated SFAPs. A simulation study based on the proposed method can help in interpreting sEMG signals detected from bi-pinnate muscles.

The analytical solution of the Poisson problem is obtained in the Fourier transformed domain and in the spatial domain by numerical inversion of the two dimensional Fourier transform. The analytical solution in the Fourier domain is valuable for two reasons: 1. Fourier inversion is numerically efficient (logarithmic cost); 2. if an a-priori knowledge of the frequency band of the 
solution is available, a proper sampling can be chosen to keep all the information (by Nyquist theorem); for sEMG the frequency band is $10-400 \mathrm{~Hz}$ in time domain, which corresponds to the spatial frequency with a relation depending on CV, Farina and Merletti [5].

As Poisson equation models different interesting physical and biological situations, the proposed method can find other applications. For example, other electrostatics problems can be addressed. Moreover, Poisson equation is obtained when a steady state solution of diffusion equations is studied (with application on heat transfer problems, pollutant dispersion, population dynamics,...). Another example of application comes from continuum mechanics theory: momentum conservation law can be reduced to Poisson equation in steady conditions, neglecting inertia, and assuming a linear constitutive relation for the stress tensor.

The proposed technique allows one to handle Poisson problems defined on a medium constituted by sub-domains with different characteristics. Such problems can be studied numerically by domain decomposition techniques. New techniques can be developed on the basis of the proposed method to interface a domain for which a numerical solution (for homogeneous Neumann and Dirichlet problems) is known with another for which an analytical solution is available.

Thus, this method could find further applications in addressing new problems in the applied sciences.

\section{References}

[1] Blok, J.H., Stegeman, D.F., and van Oosterom, A., Three-layer volume conductor model and software package for applications in surface electromyography, Ann. Biomed. Eng., 30, pp. 566-577, 2002.

[2] J. Clark, R. Plonsey, The extracellular potential field of the single active nerve fiber in a volume conductor, Biophys. Journ., vol. 8, pp. 842-64, 1968

[3] Dimitrov, G.V., Disselhorst-Klug, C., Dimitrova, N.A., Schulte, E. and Rau G., Simulation analysis of the ability of different types of multielectrodes to increase selectivity of detection and to reduce cross-talk, J. Electromyogr. Kinesiol.,13, pp. 125-38, 2003.

[4] Dimitrova N.A. and Dimitrov G.V., Interpretation of EMG changes with fatigue: facts, pitfalls, and fallacies, J. Electromyogr. Kinesiol., 13, pp. 13-36, 2003.

[5] Farina D. and Merletti R., A novel approach for precise simulation of the EMG signal detected by surface electrodes, IEEE Trans. Biomed. Eng., 48, pp. 637-646, 2001

[6] Farina D., Merletti R., Methods for estimating muscle fiber conduction velocity from surface electromyographic signals, Med. Biol. Eng. Comput., vol. 42, pp. 432-445, 2004

[7] Farina D., Mesin L., Martina S., Merletti R., Comparison of spatial filter selectivity in surface myoelectric signal detection - Influence of the 
volume conductor model, Medical \& Biological Engineering \& Computing, 42(1):114-20, 2004

[8] Farina D., Mesin L., Martina S., Merletti R., A new surface EMG generation model with multi-layer cylindrical description of the volume conductor, IEEE Transactions on Biomedical Engineering, 51(3), 415-426, 2004

[9] Farina D., Mesin L., Martina S., Advances in surface EMG signal simulation with analytical and numerical descriptions of the volume conductor, Medical \& Biological Engineering \& Computing, 42, 467-476, 2004

[10] Fuglevand A.J., Winter D. A., Patla A.E., Models of Recruitment and Rate Coding Organization in Motor-Unit Pools, Journal of Neurophysiology, v. 70, n. 6, pp. 2470-2486, 1993.

[11] Gootzen, T.H., Stegeman, D.F. and Van Oosterom, A., Finite limb dimensions and finite muscle length in a model for the generation of electromyographic signals, Electroenc. Clin. Neurophysiol., 81, pp. 152-162, 1991

[12] Heringa A., Stegeman D.F., Uijen G.J., de Weerd J.P., Solution methods of electrical field problems in physiology, IEEE Trans. Biomed. Eng., vol. 29, pp. 34-42, 1982

[13] Kleine, B.U. Stegeman D.F., Mund D. and Anders C., Influence of motoneuron firing synchronization on SEMG characteristics in dependence of electrode position, J. Appl. Physiol., 91, pp. 1588-99, 2001

[14] Lowery, M.M., Stoykov, N.S., Taflove, A. and Kuiken, T.A. A multiplelayer finite-element model of the surface EMG signal, IEEE Trans. Biomed. Eng., 49, pp. 446-454, 2002

[15] McGill, K. and Huynh, A., A model of the surface recorded motor unit action potential, Proc. 10th Annu. Int. IEEE Conf. Eng. In Med. Biol., pp. 1697-1699, 1988

[16] Merletti, R., Lo Conte, L., Avignone, E. and Guglielminotti, P., Modelling of surface myoelectric signals - part I: model implementation, IEEE Trans. Biomed. Eng., 46, pp. 810-820, 1999

[17] Merletti, R., Roy, S.H., Kupa, E., Roatta, S. and Granata, A., Modeling of surface myoelectric signals--Part II: Model-based signal interpretation, IEEE Trans. Biomed. Eng., 46, pp. 821-9, 1999

[18] Mesin L., Farina D., Simulation of surface EMG signals generated by muscle tissues with in-homogeneity due to fiber pinnation, IEEE Trans Biomed Eng. Sep;51(9):1521-9, 2004.

[19] Mesin L., Farina D., A model for surface EMG generation in volume conductors with spherical in-homogeneities, IEEE Transactions on Biomedical Engineering, in press, 2005

[20] Mesin L., Farina D., An analytical model for surface EMG generation in volume conductors with smooth conductivity variations, submitted to IEEE Transactions on Biomedical Engineering, 2005 
[21] Mesin L., Joubert M., Hanekom T., Merletti R., Farina D., A Finite Element Model for Describing the Effect of Muscle Shortening on Surface EMG, submitted to IEEE Transactions on Biomedical Engineering, 2005

[22] Roeleveld, K., Blok, J.H., Stegeman, D.F. and van Oosterom, A., Volume conduction models for surface EMG; confrontation with measurements, J. Electromyograph. Kinesiol., 7, pp. 221-232, 1997

[23] Schneider, J., Silny, J. and Rau, G., Influence of tissue inhomogeneities on non-invasive muscle fiber conduction velocity measurements investigated by physical and numerical modeling, IEEE Trans. on Biomed. Eng., 38, pp. 851-860, 1991

[24] Stegeman, D.F., Blok, J.H., Hermens, H.J. and Roeleveld, K., Surface EMG models: properties and applications, J. Electromyograph. Kinesiol., 10, pp. 313-326, 2000

[25] van Oosterom, A., Principles in inverse electrophysiological modeling, 6th Deliverable of the SENIAM project, pp. 37-44, 1998 